\title{
Identification of four novel QTL linked to the metabolic syndrome in the Berlin Fat Mouse
}

\author{
Manuel Delpero ${ }^{1}$, Danny Arends (DD ${ }^{1}$, Maximilian Sprechert ${ }^{1}$, Florian Krause ${ }^{1}$, Oliver Kluth ${ }^{2,3}$, Annette Schürmann (D) ${ }^{2,3,4}$, \\ Gudrun A. Brockmann ${ }^{1}$ and Deike Hesse (D) ${ }^{1 凶}$
}

(c) The Author(s) 2021

BACKGROUND: The Berlin Fat Mouse Inbred line (BFMI) is a model for obesity and the metabolic syndrome. This study aimed to identify genetic variants associated with impaired glucose metabolism using the obese lines BFMI861-S1 and BFMI861-S2, which are genetically closely related, but differ in several traits. BFMI861-S1 is insulin resistant and stores ectopic fat in the liver, whereas BFMI861-S2 is insulin sensitive.

METHODS: In generation 10, 397 males of an advanced intercross line (AIL) BFMI861-S1 × BFMI861-S2 were challenged with a highfat, high-carbohydrate diet and phenotyped over 25 weeks. QTL-analysis was performed after selective genotyping of 200 mice using the GigaMUGA Genotyping Array. Additional 197 males were genotyped for 7 top SNPs in QTL regions. For the prioritization of positional candidate genes whole genome sequencing and gene expression data of the parental lines were used.

RESULTS: Overlapping QTL for gonadal adipose tissue weight and blood glucose concentration were detected on chromosome (Chr) 3 (95.8-100.1 Mb), and for gonadal adipose tissue weight, liver weight, and blood glucose concentration on Chr 17 (9.5-26.1 Mb). Causal modeling suggested for Chr 3-QTL direct effects on adipose tissue weight, but indirect effects on blood glucose concentration. Direct effects on adipose tissue weight, liver weight, and blood glucose concentration were suggested for Chr 17-QTL. Prioritized positional candidate genes for the identified QTL were Notch2 and Fmo5 (Chr 3) and Plg and Acat2 (Chr 17). Two additional QTL were detected for gonadal adipose tissue weight on Chr 15 (67.9-74.6 Mb) and for body weight on Chr 16 (3.9-21.4 Mb).

CONCLUSIONS: QTL mapping together with a detailed prioritization approach allowed us to identify candidate genes associated with traits of the metabolic syndrome. In addition, we provided evidence for direct and indirect genetic effects on blood glucose concentration in the insulin-resistant mouse line BFMI861-S1.

International Journal of Obesity (2022) 46:307-315; https://doi.org/10.1038/s41366-021-00991-3

\section{INTRODUCTION}

The metabolic syndrome is defined as a metabolic abnormality that leads to high body weight, ectopic fat storage, insulin resistance, high blood pressure, and chronic low-grade inflammation [1]. Heritability estimates for each trait of the metabolic syndrome are high with some estimates exceeding 50\% [2]. Nevertheless, genome-wide association studies on body mass index and other traits of the metabolic syndrome identified loci, that combined, account for only $1-7 \%$ of the variance in the examined population [2]. Therefore, studies on different populations are needed to identify additional causal genes to better understand their direct and interaction effects contributing to the metabolic syndrome.

The goal of the current study was to identify genetic factors contributing to obesity and glucose homeostasis in the Berlin Fat Mouse. Originally, the Berlin Fat Mouse population was selected for juvenile obesity. After 58 generations of selection, different
Berlin Fat Mouse Inbred (BFMI) lines were generated through repeated brother-sister mating [3]. In a cross between the most obese inbred line BFMI860 and the lean control line C57BL/6NCrl, we have previously identified a recessive genetic defect at a locus on chromosome (Chr) 3 accounting for $40 \%$ of the variance in adipose tissue weight at 6 weeks $[4,5]$. This juvenile obesity locus (jObes 1 ) is fixed in all BFMI sublines.

In the current study, we used the inbred lines BFMI861-S1 (S1) and BFMI861-S2 (S2). S1 and S2 are sublines created from the BFMI860, as such the BFMI860 is the predecessor of the S1 and S2. The S1 and S2 lines were conspicuously different with respect to metabolic traits [6]. In particular, the S1 line showed high body weight, hepatic fat storage, low insulin sensitivity, and impaired glucose tolerance. In contrast, S2 is insulin sensitive despite being obese [6]. This observation was particularly interesting since these two lines were derived from one parental line that was divided into two sub-lines

\footnotetext{
${ }^{1}$ Albrecht Daniel Thaer-Institut für Agrar- und Gartenbauwissenschaften, Humboldt-Universität zu Berlin, Berlin, Germany. ${ }^{2}$ Department für Experimentelle Diabetologie, Deutsches Institut für Ernährungsforschung Potsdam-Rehbrücke (DIfE), Nuthetal, Germany. ${ }^{3}$ German Center for Diabetes Research (DZD), München-Neuherberg, Germany. ${ }^{4}$ University of Potsdam, Institute of Nutritional Science, Potsdam, Germany. ${ }^{\bowtie}$ email: deike.hesse-wilting@hu-berlin.de
} 
only after four generations of inbreeding. Therefore, these two lines are genetically highly similar, and the remaining genetic diversity is responsible for phenotypic differences. To identify genetic loci accounting for the observed obesity, and glucose homeostasis in S1 mice, we performed a quantitative trait locus (QTL) mapping study in an advanced intercross line (AIL) which was generated from an initial cross between the BFMI861 lines S1 and S2. In this study, all AlL mice were challenged with a high-fat, high-carbohydrate diet.

\section{MATERIAL AND METHODS Mouse population}

We used male mice of the parental mouse lines BFMI861-S1 (S1) and BFMI861-S2 (S2) and generation 10 of an AlL population. The AlL population was generated from an initial cross between a BFMI861-S1 (S1) male and a BFMI861-S2 (S2) female followed by repeated random mating in every generation. For randomization of mating pairs, the program RandoMate [7] was used. The BFMI861 lines S1 and S2 were generated as described in Heise et al. [6].

\section{Animal husbandry}

All experimental treatments of mice were approved by the German Animal Welfare Authorities (approval no. G0235/17). Mice were kept under conventional conditions with a 12:12 h light-dark cycle (lights on at $0600 \mathrm{~h}$ ) and at a temperature of $22 \pm 2{ }^{\circ} \mathrm{C}$. Mice had ad libitum access to food and water.

\section{Experiment and phenotyping}

Data from parental strains S1 and S2 were collected at 20 weeks on a standard diet containing $16.7 \mathrm{MJ} / \mathrm{kg}$ of metabolizable energy, $11 \%$ from fat, $26 \%$ from protein, and $53 \%$ from carbohydrates (V1534-000, ssniff EF R/ $\mathrm{M}$; Ssniff Spezialdiäten $\mathrm{GmbH}$, Soest, Germany) and blood glucose was measured at 25 weeks after 5 weeks exposure to a high-fat, highcarbohydrate diet containing $21.9 \mathrm{MJ} / \mathrm{kg}$ of metabolizable energy, $28 \%$ from fat, $20 \%$ from protein and $40 \%$ from carbohydrates [8].

To emphasize the difference in glucose homeostasis, all AlL animals were challenged with a dietary regime that provides a gluco-lipotoxic environment for the $\beta$-cells and thereby provokes differences in $\beta$-cell resilience [9]. This dietary regime challenge was undertaken to provoke differences in the phenotypes studied. Until the age of 20 weeks, AlL mice were fed the rodent standard diet. In weeks 21 and 22, mice were fed a high-fat, low-carbohydrate diet, containing $16.9 \mathrm{MJ} / \mathrm{kg}$ of metabolizable energy, 34\% from fat, $19 \%$ from protein, and 47\% from carbohydrates (C1057; Altromin Spezialfutter GmbH \& Co. KG, Lage, Germany) to increase obesity but to protect $\beta$-cells. Afterward, animals were fed for 3 weeks a high-fat, high-carbohydrate diet containing $21.9 \mathrm{MJ} / \mathrm{kg}$ of metabolizable energy, $28 \%$ from fat, $20 \%$ from protein, and $40 \%$ from carbohydrates [8] to challenge $\beta$-cells with carbohydrates and thereby increase differences in glucose metabolism.

AlL mice were phenotyped between the age of 3 (after weaning) and 25 weeks. Body mass was recorded weekly. To investigate glucose metabolism, an oral glucose tolerance test (oGTT) was performed in week 18 and an intraperitoneal insulin tolerance test (ITT) in week 20 as described before [6]. The area under the curve (AUC) for blood glucose concentration of oGTT and ITT was calculated. At 25 weeks, final blood glucose concentration was recorded after fasting for two hours. Afterward, mice were anesthetized with isoflurane and sacrificed [10]. Gonadal adipose tissue (GonAT), subcutaneous adipose tissue, liver, and skeletal muscle (quadriceps) were dissected and weighed. Tissues were collected in liquid nitrogen and stored at $-80^{\circ} \mathrm{C}$. Protein content and triglycerides of homogenized liver samples were determined as described in Hesse et al. [11].

Outliers, defined as individuals who have a measurement that deviates from the population mean by more than four standard deviations (SD), were removed from the data. Pearson's correlation coefficients were calculated between normal distributed traits. For non-normal distributed traits, Spearman's correlation coefficients were calculated.

\section{Genotyping}

Out of the 397 males that were phenotyped, selective genotyping was performed; 200 mice representing the two tails of the phenotypic distributions of gonadal adipose tissue weight and liver weight were selected for genotyping with the Giga Mouse Universal Genotyping Array (GigaMUGA; Illumina, San Diego, CA, USA) [12]. Genotyping was done at Neogen GeneSeek (Lincoln, NE, USA). Due to high genetic similarity of the parental lines S1 and S2 of the AlL population, only 5215 out of 143,259 SNPs on the array were informative and passed the quality control (supplementary Fig. 1, supplementary File 1).

Remaining 197 males of the AlL population were genotyped for 7 top markers. For these markers, KASP genotyping assays were developed as described previously [13] (supplementary File 2). The additional animals were genotyped to counteract any bias in the estimates of allele effect sizes introduced by selective genotyping.

\section{QTL mapping}

QTL mapping was performed in two steps: First, a QTL scan was performed using the 200 males that were genotyped with the GigaMUGA Array. Afterward, a final QTL scan was performed including all animals (genotyped by GigaMUGA and KASP).

Covariates (subfamily and litter size) were investigated for a significant influence on each phenotype. Covariate analysis showed that litter size significantly influenced liver weight $(p<0.02)$, as such, litter size was added as a covariate to the model when QTL mapping liver weight. No other significant covariates were found. Using pedigree information of the AlL population, we tested the sub-family effect on the phenotype, but no significant influence was found (code available upon request).

To confirm that QTL mapping models are valid, residuals of the models were tested for normality using a Shapiro-Wilk test. If residuals were found to not be normally distributed, a nonparametric Kruskal-Wallis one-way analysis of variance was performed to validate the top marker.

The number of independent statistical tests was estimated by simpleM [14] which determined the number of independent tests to be 849 (window size $=820, \mathrm{mEff}=849$ ). Afterwards Bonferroni correction for multiple testing correction [15] was performed using the number of independent SNPs as determined by simpleM. $P$ values were converted to LOD scores, using LOD $=-\log 10$ ( $p$ value). LOD scores above 4.9 and 4.2 were deemed to be genome-wide highly significant and significant, respectively. The $95 \%$ confidence interval of a QTL was determined by a 1.5 LOD drop from the top SNP position [16]. Start and end positions were defined as the first SNP upstream or downstream of the 1.5 LODdrop confidence interval.

\section{Causal modeling}

In case of an overlapping QTL between multiple traits, we applied pairwise causal modeling as previously described $[17,18]$. In short, when a common QTL is found for two (or more traits), we model the effect of the QTL on these traits in a pairwise manner. Causal modeling was performed by comparing the independent model (QTL directly affects both $\mathrm{T} 1$ and $\mathrm{T} 2$ ) with the causal/ reactive model (QTL directly affects T1 which in turn affects T2). Of course, it can happen that none of the models fit the data satisfactory, we then assume causality is undetermined. Statistical models used for causal modeling are described in more detail in supplementary File 3.

Direct QTL effects are defined as caused by a QTL which directly affects the variability of both traits (independent model fits best, QTL directly affects T1 and T2). Indirect effects were defined as effects on a trait (T1) caused by a QTL through another trait (T2) (causal model fits best). In this case, the QTL is defined as having a direct effect on $\mathrm{T} 2$, and an indirect effect on T1. Causal modeling to determine direct and indirect effects of QTL on traits was performed for GonAT weight, liver weight, and blood glucose concentration on Gatlgq and for GonAT weight and blood glucose concentration on Gatq1.

\section{Whole-genome sequencing}

The two parental lines of the AlL (S1 and S2) were paired-end sequenced using the "Illumina HiSeq" (Illumina) platform. Obtained DNA reads were trimmed and aligned to the mouse genome (MM10, GRCm38.p3), sequence variants were called using BCFtools and annotated using the Ensembl Variant Effect Predictor (VEP) $[19,20]$. VEP provided information on the position of SNPs within known motifs such as promoters, regulatory sites, and protein domains. DNA sequencing data were deposited at the NCBI Sequence Read Archive under BioProject ID: PRJNA717237.

\section{Gene expression analysis}

RNA was isolated from gonadal adipose tissue (S1: $n=7, \mathrm{~S} 2: n=8)$, liver (S1: $n=7, \mathrm{~S} 2: n=8)$ and skeletal muscle (S1: $n=7, \mathrm{~S} 2: n=8)$ of males of the parental lines S1 and S2 at 10 weeks. Pancreatic islets (S1: $n=6, \mathrm{~S} 2$ : $n=6$ ) were isolated as described in Gotoh et al. [21] and RNA was extracted as described [10]. Gene expression was measured with the 


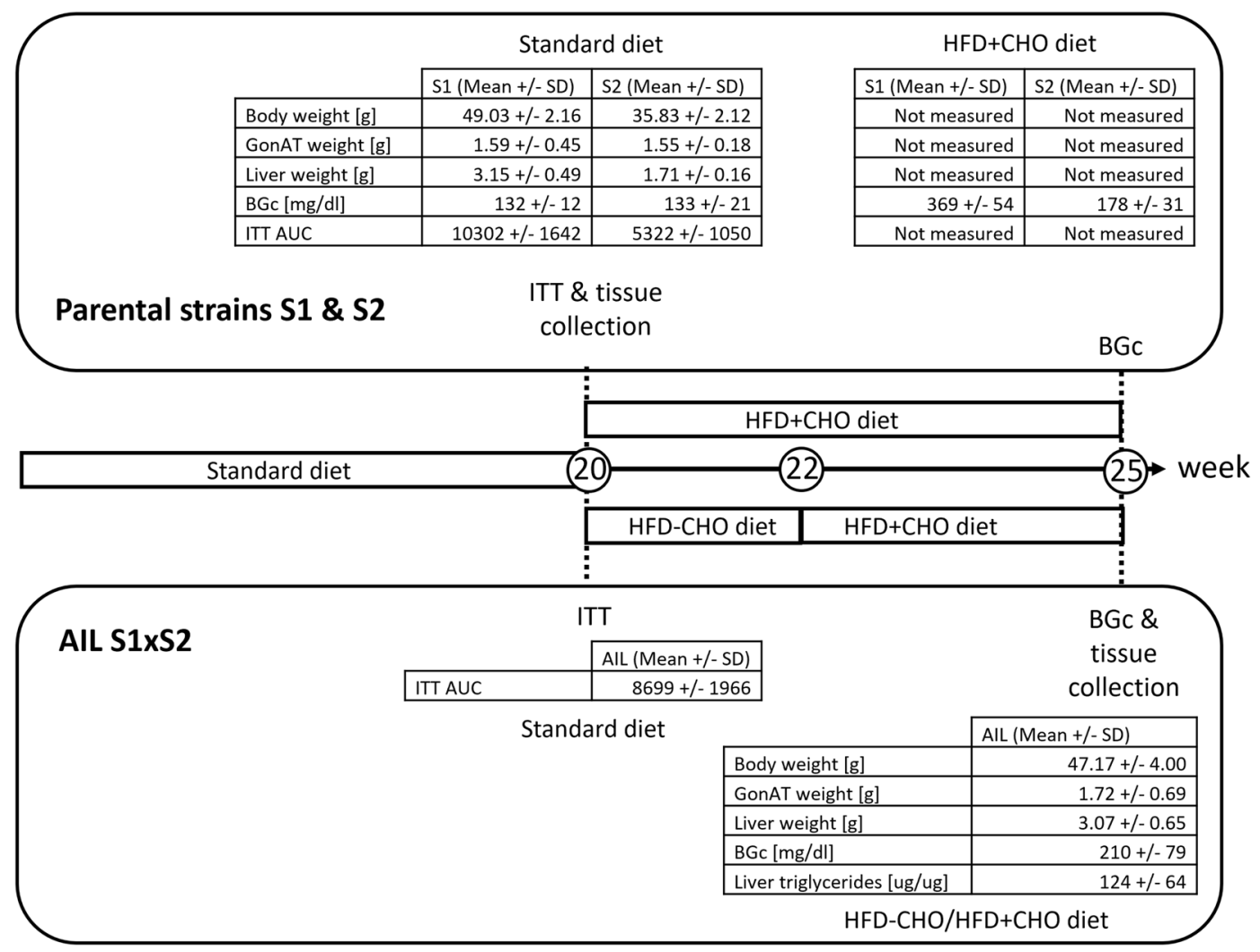

Fig. 1 Response of parental lines S1 and S2, and AIL males to high-fat, high-carbohydrate diet. GonAT, gonadal adipose tissue; BGc, blood glucose concentration; ITT, insulin tolerance test; AUC, area under the curve; HFD-CHO, high-fat/low-carbohydrate diet; HFD + CHO, high-fat/ high-carbohydrate diet.

Clariom S assay for mouse (Thermo Fisher Scientific) using service (ATLAS Biolabs, Berlin, Germany). The intensity values of the arrays were transformed to the logarithm of base 2 and quantile normalized for each tissue separately. To test for expression differences between S1 and S2 mice, t-tests were performed for each probe on the array in each tissue. Benjamini-Hochberg correction was applied for multiple testing. $\mathrm{R}$ was used for statistical analysis and graphical presentation [22]. Quantitative real-time PCR was performed as described in Heise et al. [6]. Relative transcript amounts were calculated using the relative quantification method (ddCT-method) [23]. Gene-specific primers are available in supplementary File 4.

\section{Candidate gene prioritization}

Genomic DNA sequences of all protein-coding positional candidate genes were downloaded using bioMART [24]. To include regulatory regions such as promoters, we considered additional 1000 base pairs from the start and end position of each gene. Monomorphic genes without sequence variants between S1 and S2 were removed from the list of positional candidate genes. All other genes were scored for potential functional effects of sequence variants, gene expression differences between $\mathrm{S} 1$ and $\mathrm{S} 2$ in gonadal adipose tissue and liver, and their contribution to KEGG pathways [25]. Coding sequence variants leading to stop gain/stop loss codons and missense mutations located in functional protein domains were awarded 3 points to the gene score. A missense variant with either a deleterious or a tolerated SIFT (Sorting Intolerant From Tolerant) value obtained 3 or 1 point, respectively. Non-coding variants were scored based on their location in potential functional sites. If a non-coding variant was located in the promoter or in a splice site, 3 points were awarded; if located in untranslated regions (UTRs), enhancers, or CTCF binding sites (involved in 3D structure of chromatin) 1 point was awarded. Genes differentially expressed in at least one tissue were awarded 2 points. Genes annotated in relevant KEGG metabolic pathways were awarded 1 point. Genes in KEGG pathways were downloaded using the R package "StarBioTrek" [26]. To find further evidence for potential causality, the highest scored candidate genes were screened for metabolic processes or diseases using Gene Ontology, public literature, and databases such as Mouse Genome Informatics and the International Mouse Phenotyping Consortium.

\section{RESULTS}

Response of parental lines S1 and S2, and AIL males to high-fat, high-carbohydrate diet

According to SNP chip data (GigaMuga), S1 and S2 animals are $96.4 \%$ genetically identical (supplementary Fig. 1b). However, with a standard diet S1 males had significantly higher body weight $(p<$ $0.001, n=10)$ and higher liver weight $(p<0.001, n=10)$ compared to S2 males at 20 weeks of age (Fig. 1). To challenge the glucose homeostasis, we fed 20 weeks-old S1 and S2 mice a high-fat, highcarbohydrate diet for 5 weeks and observed extreme high blood glucose concentration in S1 males ( $369 \pm 54 \mathrm{mg} / \mathrm{dl}$ ) compared to S2 males (178 $\pm 31 \mathrm{mg} / \mathrm{dl}$ ) (Fig. 1). To elucidate the genetic impact on the response to this challenge the AlL population was exposed to a gluco-lipotoxic environment provoking differences in $\beta$-cell resilience [9]. Therefore, the diet was switched at 20 weeks from a standard diet to a lipotoxic high-fat, low-carbohydrate diet (two weeks) to increase obesity, followed by a gluco-lipotoxic high-fat, high-carbohydrate diet for additional three weeks to challenge $\beta$-cells. At 25 weeks AlL mice showed an average blood glucose concentration of $210 \pm 79 \mathrm{mg} / \mathrm{dl}$. In addition, gonadal adipose tissue weight was $1.72 \pm 0.69 \mathrm{~g}$ and liver weight was $3.07 \pm 0.65 \mathrm{~g}$ on average. Liver triglycerides/protein content was $124 \pm 64 \mu \mathrm{g} / \mu \mathrm{g}$, and body weight was $47.17 \pm 4.00 \mathrm{~g}$ on average (Fig. 1).

\section{Correlation between traits}

In metabolically healthy individuals of our AIL we would expect high body weights associated with high adipose tissue weight, unchanged liver weight, and normal glucose clearance. In contrast, the correlation analysis showed no correlation of body weight with gonadal adipose tissue weight $(r=0.02, p=0.65)$, but a positive correlation with liver weight $(r=0.65, p=2.20 \mathrm{E}-16)$ and liver triglycerides ( $r=0.39, p=1.94 \mathrm{E}-15)$ (Table 1) (Supplementary Fig. 2). Moreover, a negative correlation was found between gonadal 
Table 1. Correlation coefficients between the collected traits in the AlL population.

\begin{tabular}{|llllll|} 
& $\begin{array}{l}\text { Liver weight } \\
(\boldsymbol{r} \text { and } \boldsymbol{P} \text { value) }\end{array}$ & $\begin{array}{l}\text { Liver triglycerides/protein } \\
(\boldsymbol{r} \text { and } \boldsymbol{P} \text { value) }\end{array}$ & $\begin{array}{l}\text { BGc } \\
(\boldsymbol{r} \text { and } \boldsymbol{P} \text { value) }\end{array}$ & $\begin{array}{l}\text { ITT AUC } \\
(\boldsymbol{r} \text { and } \boldsymbol{P} \text { value) }\end{array}$ & $\begin{array}{l}\text { Body weight } \\
(\boldsymbol{r} \text { and } \boldsymbol{P} \text { value) }\end{array}$ \\
\hline GonAT weight & $-0.47,2.20 \mathrm{E}-16$ & $-0.33,2.88 \mathrm{E}-11$ & $-0.59,2.20 \mathrm{E}-16$ & $-0.41,2.20 \mathrm{E}-16$ & $0.02,0.65$ \\
\hline Liver weight & & $0.55,2.20 \mathrm{E}-16$ & $0.71,2.20 \mathrm{E}-16$ & $0.27,3.98 \mathrm{E}-08$ & $0.65,2.20 \mathrm{E}-16$ \\
\hline Liver triglycerides/protein & & $0.45,2.20 \mathrm{E}-16$ & $0.28,1.41 \mathrm{E}-08$ & $0.39,1.94 \mathrm{E}-15$ \\
\hline BGC & & & $0.34,9.55 \mathrm{E}-13$ & $0.31,2.55 \mathrm{E}-10$ \\
\hline ITT AUC & & & $0.21,2.56 \mathrm{E}-05$ \\
\hline
\end{tabular}

GonAT, gonadal adipose tissue; BGc, blood glucose concentration; ITT, insulin tolerance test; AUC, area under the curve.

Table 2. Position and effects of QTL identified in the AlL population of 397 mice.

Traits

\begin{tabular}{|c|c|c|c|c|}
\hline & QTL name & Chr & StartPos & TopPos \\
\hline GonAT weight [g] & Gatlgq & 17 & 9483181 & 25258903 \\
\hline Liver weight [g] & & 17 & 9483181 & 25258903 \\
\hline BGc [mg/dl] & & 17 & 11934634 & 25258903 \\
\hline GonAT weight [g] & Gatq1 & 3 & 95763020 & 98196163 \\
\hline BGc [mg/dl] & & 3 & 95763020 & 98196163 \\
\hline Body weight [g] & Bwq26 & 16 & 3892297 & 11120784 \\
\hline GonAT weight [q] & Gatq2 & 15 & 67855285 & 68461862 \\
\hline
\end{tabular}

QTL confidence interval

StopPos

25391933

25391933

26054796

100780367

100543098

21355904

74582319

$\operatorname{LOD}(\mathrm{BH})$

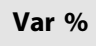

\section{Mean S1}

$\begin{array}{ll}\Delta \text { Mean } & \Delta \text { Mean } \\ \text { S1-HET } & \text { S1-S2 }\end{array}$

GonAT gonadal adipose tissue; BGc, blood glucose concentration; QTL quantitative trait locus; Chr chromosome number; StartPos, TopPos, and StopPos, position of the start of the QTL confidence interval, position of the SNP with the highest LOD score, and position of the end of the QTL confidence interval in base pairs, respectively; Positions are given according to the Mouse Genome Version MM10, GRCm38.p3. SNP, single-nucleotide polymorphism. The confidence interval gives the 1.5 LOD drop region of the top SNP position. A LOD score above 4.9 was deemed to be "genome-wide highly significant" and above 4.2 was deemed "genome-wide significant"; BH, Bonferroni Benjamini-Hochberg correction; LOD, logarithm (base 10) of odds; Var \%, percentage of total variance explained; The $\Delta$ Mean columns show the phenotypic difference between homozygous S1 and heterozygous BFMI animals (S1-HET) and the difference between homozygous S1 and S2 animals (S1-S2).

adipose tissue and liver weight ( $r=-0.47, p=2.2 \mathrm{E}-16)$, and gonadal adipose tissue weight and liver triglycerides $(r=-0.33, p=2.88 \mathrm{E}$ $-11)$. Low adipose tissue weight together with high body weight and high liver weight was also associated with a large area under the curve for the blood glucose concentration in the ITT $(r=-0.41$, $p=2.2 \mathrm{E}-16)$ and high blood glucose concentration $(r=-0.59, p=$ 2.2E-16). Consistent with the negative correlation coefficients for adipose tissue weights and the other parameters, positive correlations were found between liver weights and the same parameters (Table 1) (supplementary Fig. 2).

\section{QTL mapping}

QTL mapping was performed for body weight, gonadal adipose tissue weight, liver weight, liver triglycerides, and blood glucose concentration at the end of the experiment and for ITT AUC at 20 weeks before the diet switch. The QTL analysis on selectively genotyped 200 AlL males revealed significant loci on Chr 3, 15, 16, and 17 (supplementary Table 1). The follow-up analysis after KASP genotyping including all 397 males confirmed all four QTL and provided true estimates for the genetic effects (Table 2).

Three significant QTL for gonadal adipose tissue weight were identified on Chr 17 (Gatlgq) at 25.25 Mb (LOD = 7.3), Chr 3 (Gatq1) at $98.19 \mathrm{Mb}(\mathrm{LOD}=6.3)$, and on Chr $15(\mathrm{Gatq} 2)$ at $68.46 \mathrm{Mb}(\mathrm{LOD}=$ 4.2). Interestingly, for these three QTL, the $S 1$ allele always decreased the amount of adipose tissue. Gatlgq had also an effect on liver weight ( $L O D=7.5$ ) which could be caused by an increased hepatic fat storage. Indeed, liver triglycerides show a significant effect on Gatlgq based on genome-wide multiple testing correction when we consider the selectively genotyping of the initial 200 selected animals ( $L O D=4.8)$. Mapping liver triglycerides using the whole population there is still an effect $(L O D=2.4)$. However, this effect does not reach the threshold for genome-wide significance $(<0.05)$ but is still suggestive $(P<0.1)$. Furthermore, Gatlgq and Gatq1 affected the blood glucose concentration (LOD Gatlgq $=8$, LOD Gatq1 = 4.2). For Gatlgq the allele of the insulin-resistant S1 line was responsible for low adipose tissue weight, elevated liver weight, higher liver triglycerides, and high blood glucose concentration. The S1-allele effects of the Gatlgq on gonadal adipose tissue weight and liver weight and on gonadal adipose tissue weight and liver triglycerides were in opposing direction, supporting the negative correlation between the traits. In contrast, for Gatq1 the S1-QTL allele decreased the adipose tissue weight, reduced the blood glucose concentration (Table 2, Fig. 2b), and was associated with faster glucose clearance in the ITT (LOD = 3.8).

A QTL for body weight was mapped on Chr 16 (Bwq26) at $11.12 \mathrm{Mb}(\mathrm{LOD}=7.1)$. At this locus, the $\mathrm{S} 1$ allele was increasing body weight (Table 2).

Since Gatlgq and Gatq1 showed pleiotropic effects on several traits, causal modeling was performed. Causal modeling of Gatlgq suggests direct effects on gonadal adipose tissue, liver weight, and blood glucose concentration. Causal modeling of Gatq1 showed a direct effect of Gatq1 on gonadal adipose tissue weight, which in turn affects blood glucose concentration.

\section{Candidate gene prioritization}

The confidence intervals of the four significant QTL contain 534 protein-coding potential candidate genes. Sixty-two genes were polymorphic between S1 and S2; 27 in Gatlgq, 27 in Gatq1, 4 in Bwq26, 4 in Gatq2. Mutations in these genes were scored for their potential functional effects on the quality or expression level of the encoded protein according to the decision tree (Fig. 3a). None of the genes carried a stop gain or stop loss 
A
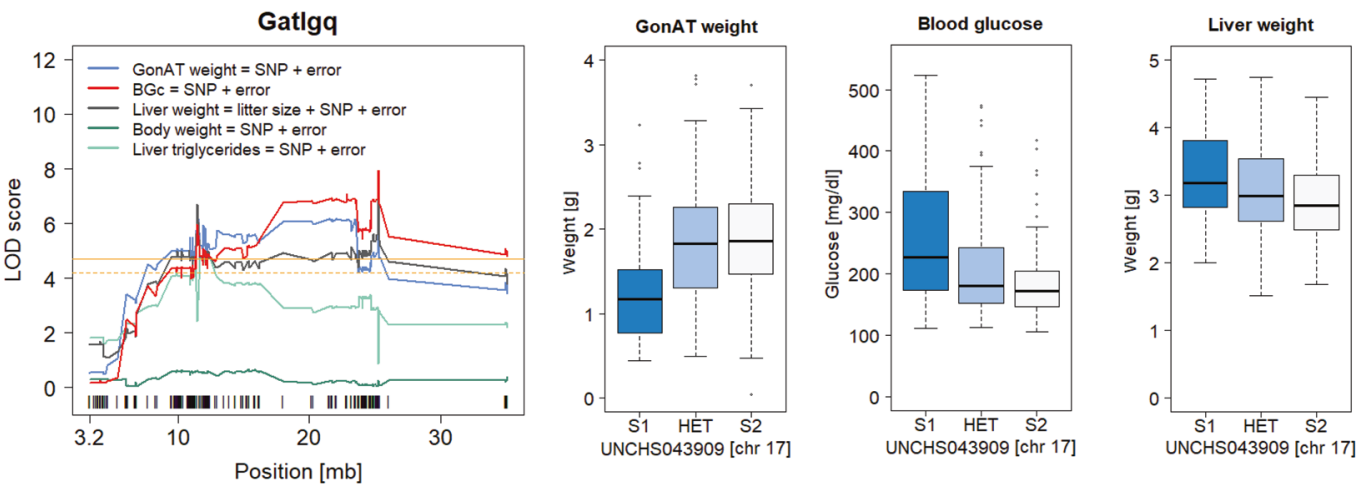

B
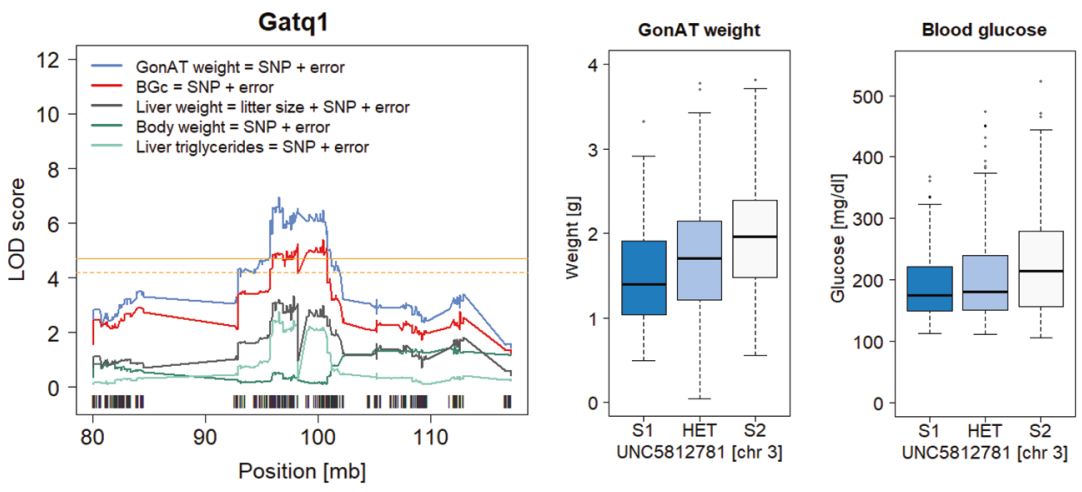

C
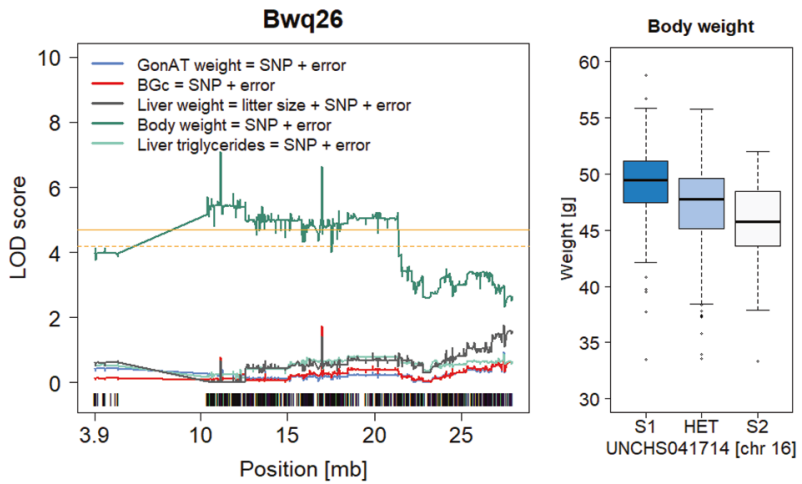

D
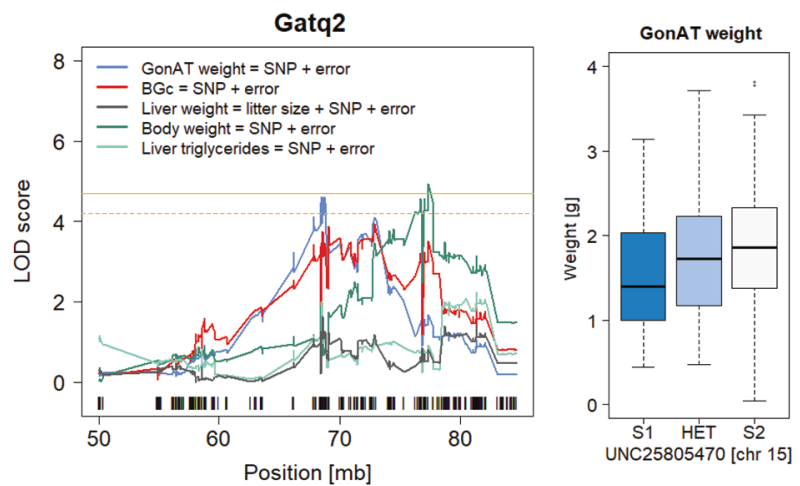

Fig. 2 Significant QTL identified in the AIL population. LOD score profiles and effect plots for top SNPs of significant traits ( $n=397$ ) for A Gatlgq, B Gatq1, C Bwq26, D Gatq2. Blue line - gonadal adipose tissue weight, black line - liver weight, red line - blood glucose concentration, dark green line - body weight, light green line - liver triglycerides. QTL, quantitative trait locus; GonAT, gonadal adipose tissue; BGc, blood glucose concentration; SNP, single-nucleotide polymorphism; Chr, chromosome number; HET, heterozygous. 

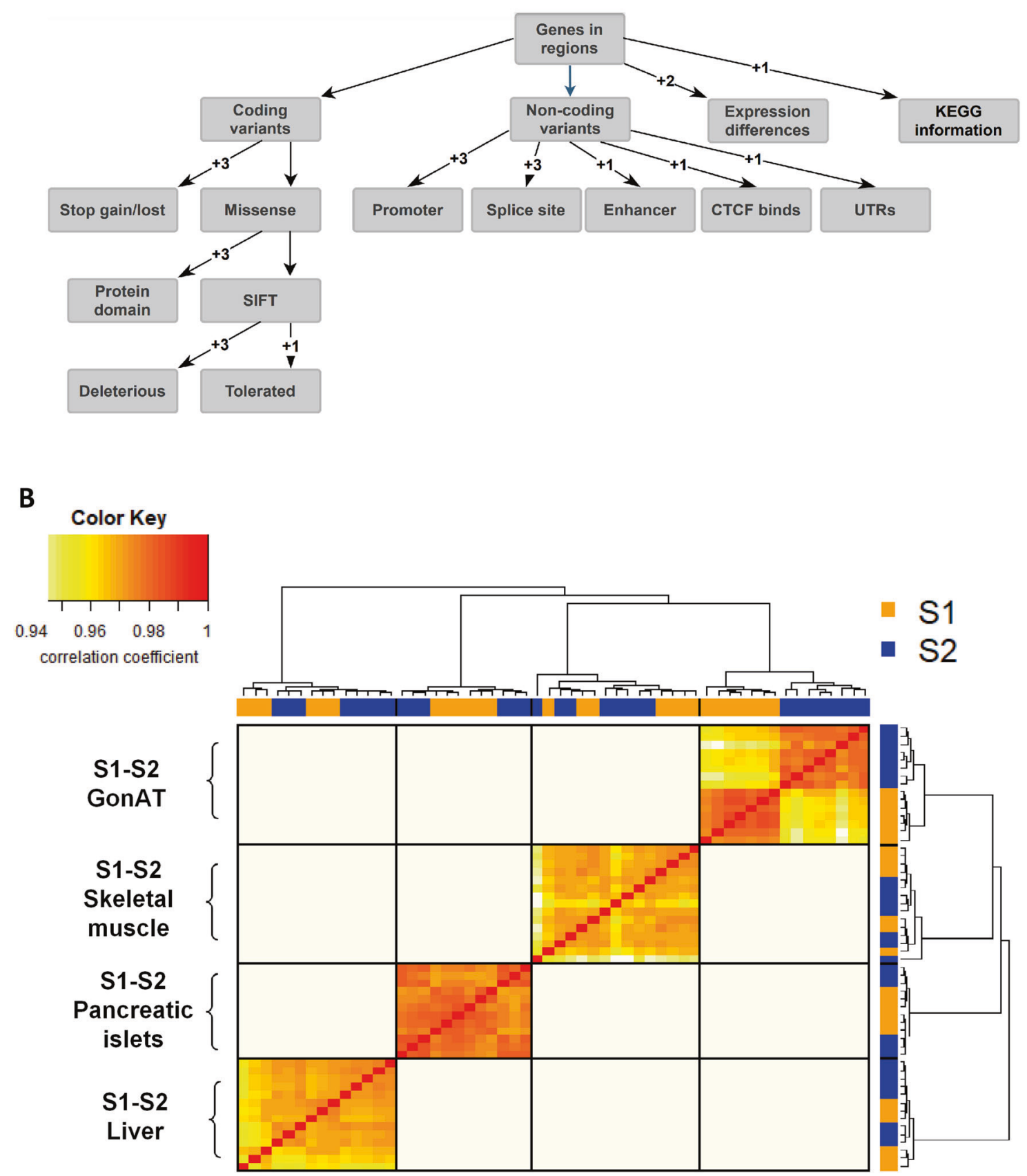

Fig. 3 Prioritization of positional candidate genes and identification of the main causal tissue. A Decision tree for prioritization of candidate genes located in a QTL region. Genes in a QTL region containing sequence variants between the parental lines S1 and S2 were ranked according to the sum of scores based on the functional annotation of coding and non-coding variants, gene expression data, and KEGG information. B Heatmap and dendrogram of microarrays gene expression data from four different tissues (gonadal adipose tissue, skeletal muscle, pancreatic islets and liver) of the parental lines (S1 and S2).

mutation. Nevertheless, different mutations influencing protein sequence or gene regulation occurred (supplementary Table 2 ). According to microarrays analysis, considering the 62 candidate genes, we found 37 genes differentially expressed between S1 and $\mathrm{S} 2$ in the gonadal adipose tissue, 8 in the liver, 8 in pancreatic islets, and 3 in skeletal muscle (Supplementary Files 5, 6,7 , and 8 , respectively). Since correlation analysis of gene expression data between all examined animals showed that mice of the same mouse line clustered together only with gene expression data of the gonadal adipose tissue (Fig. 3b), gonadal adipose tissue is suggested as the main tissue contributing to obesity and glucose homeostasis in the S1 line.

Genes with the highest and second-highest score in every QTL confidence interval were regarded as top candidates (Table 3). Differences in the expression of the top candidate genes for each QTL were confirmed by quantitative real-time PCR in both gonadal adipose tissue and liver (supplementary Fig. 3). Plg (plasminogen) and Acat2 (acetyl-CoA acetyltransferase 2, cytosolic) are the top candidates in Gatlgq. Plg was not differentially expressed but contains one tolerated missense variant in the low-complexity region, one SNP in 


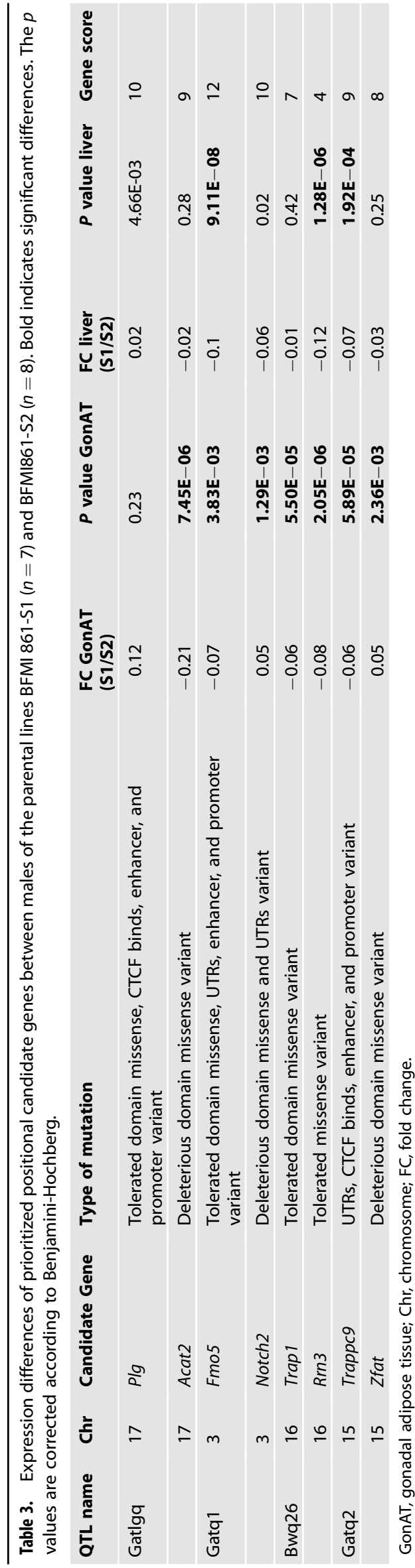

the promoter, and additional SNPs in enhancers and CTCF binding sites in mice of the S1 line. Acat2 was lower expressed in gonadal adipose tissue of S1 mice $(p=7.45 \mathrm{E}-06)$ and carries a deleterious missense variant in the thiolase, N-terminal domain. In Gatq 1 Fmo5 (flavin-containing monooxygenase 5) and Notch2 (notch homolog 2) were prioritized. Fmo5 was lower expressed in gonadal adipose tissue $(p=3.83 \mathrm{E}-03)$ and liver (9.11E-08) of S1 versus S2 mice. The Fmo5 gene in $\mathrm{S} 1$ mice carries one tolerated missense variant in the FMO-like domain, SNPs in the promoter, and additional SNPs in enhancers and untranslated regions. Notch2 ( $p=1.29 \mathrm{E}-03)$ was higher expressed in gonadal adipose tissue of S1 mice and carries one deleterious missense variant located in the EGF-like domain plus SNPs in untranslated regions in S1 mice. Trap1 (TNF receptor-associated protein 1) and Rrn3 (RRN3 homolog, RNA polymerase I transcription factor) ranked highest in Bwq26. Both candidate genes Trap1 ( $p=$ 5.50E-05) and Rrn3 ( $p=2.05 \mathrm{E}-06)$ were lower expressed in gonadal adipose tissue, and Rrn3 was additionally significantly lower expressed in the liver $(p=1.28 \mathrm{E}-06)$ of S1 mice. Both Trap1 and Rrn3 carry one tolerated missense variant. For Gatq2 Trappc9 (trafficking protein particle complex subunit 9) and Zfat (zinc finger and AT hook domain containing) ranged as top candidates. Trappc9 was lower expressed in $\mathrm{S} 1$ versus S2 mice in both gonadal adipose tissue $(p=5.89 \mathrm{E}-05)$ and liver $(p=1.92 \mathrm{E}-04)$. Trappc9 possesses variants in UTRs, CTCF binding sites, enhancer, and promoter. Zfat was higher expressed $(p=2.36 \mathrm{E}-$ 03 ) in gonadal adipose tissue of S1 mice and it carries one deleterious missense variant in the low-complexity region in S1 mice.

\section{DISCUSSION AND CONCLUSIONS}

To better understand the differences in insulin sensitivity in two sublines of the Berlin Fat Mouse independent of a major obesity QTL on Chr 3 (jObes1) and to unravel the genetic architecture underlying the observed aspects of the metabolic syndrome we investigated an advanced intercross population of the BFMI861 mouse lines S1 and S2. Besides being genetically closely related and sharing the known obesity locus on Chr3 [5], the two parental mouse lines differ extremely in their metabolic phenotype. The mouse line S1 showed clear features of the metabolic syndrome while S2 was also obese, but had normal glucose homeostasis even under a high-fat, highcarbohydrate diet feeding. The extreme phenotypic data propose the examined mouse lines as an excellent model for studying the genetic determinants of traits of the metabolic syndrome. Due to the random mixture of the genomes of the BFMI861-S1 and -S2 lines, the AlL individuals showed a wide range of phenotypes. Different from expectations in metabolically healthy individuals, we found no correlation between body weight and gonadal adipose tissue weight and negative correlations between gonadal adipose tissue weight and all other traits in AlL males, while liver weight was positively correlated with all other traits. These findings indicate ectopic fat storage in the liver which was indeed confirmed by the assessment of hepatic triglycerides in our AlL. Hepatic fat storage is also present in individuals of the S1 line [6]. Ectopic fat storage in the liver instead of storage in the adipose tissue as the major fat storage organ has been reported repeatedly as causal defect for later impaired glucose clearance $[27,28]$. Therefore, we suggest this shift as the likely driver for impaired glucose homeostasis in our mouse model. Gene expression data further supported the assumption of impaired adipose tissue function being causal for the observed phenotypes of the metabolic syndrome in S1 mice. For example, we found distinguished clusters of differentially expressed genes in gonadal adipose tissue between S1 and S2 animals but not in liver, muscle, and pancreatic islets.

The overlap of QTL effects in some regions is consistent with the correlations that we found between the affected traits. For Gatlgq, the S1 allele reduces adipose tissue weight and increases the liver weight and hepatic fat content. Moreover, by shifting fat storage from adipose tissue to ectopic storage in the liver blood glucose concentration is increased. In contrast, the S1 allele on 
Gatq1 contributes to lower adipose tissue weight, lower blood glucose concentration, and increased insulin sensitivity.

To disentangle the direct and indirect genetic effects of the different QTL, we performed causal modeling. Using causal inference, we were able to provide evidence that out of the two QTL associated with blood glucose concentration in our population, likely only Gatlgq has a direct influence on blood glucose concentration. The second QTL, Gatq1, influences blood glucose concentration indirectly through the regulation of fat storage in adipose tissue, whose weight is directly affected by this QTL. A possible explanation for the discrepancy in the correlation of adipose tissue weight to blood glucose concentration of the two QTL could be that a reduced adipose tissue mass via the S1 allele of Gatlgq could indicate a shift towards ectopic fat storage which is reflected in elevated liver weight and liver triglycerides and thereby contributes to higher blood glucose concentration. In contrast, Gatq1 and Gatq2 could harbor S1 alleles protecting against obesity resulting in lower adipose tissue weight which for Gatq1 is accompanied by lower blood glucose concentration. However, the overall phenotype of the insulin-resistant S1 line appears to be driven mainly by the larger effects of Gatlgq. These findings provide strong evidence for the importance of direct genetic effects on adipose tissue, which indirectly contribute to the etiology of impaired glucose homeostasis.

The AlL population used in this study has the advantage of having a high mapping resolution with respect to the call of positional candidate genes. Because the examined AlL accumulates chromosomal recombination over ten generations, the physical length of the QTL regions is relatively short and thereby the number of positional candidate genes low. In our study, the number of positional candidate genes could be further reduced because long chromosomal stretches are identical between the closely related mouse lines S1 and S2 and, therefore, genes in these regions are not polymorphic and can be excluded from further studies, resulting in 62 out of 534 proteincoding genes as positional candidate genes.

In our prioritization approach, Plg and Acat2 are the top candidate genes for direct effects of Gatlgq on gonadal adipose tissue weight, liver weight, and blood glucose concentration. Plg possesses one tolerated missense variant in the low-complexity region of the protein in $\mathrm{S} 1$ mice, a region that is significant for the functionality of this protein [29]. Plg-knockout mice have lower amounts of adipose tissue [30]. During cell differentiation plasminogen binding is increasing in 3T3 cells and isolated adipocytes suggesting a role in adipose tissue development [31]. In humans, Plg was reported to be relevant for the development of insulin resistance and diabetes [32-34]. Thus, Plg could be causal for the impaired glucose homeostasis by modifying adipose tissue in S1 mice. Acat2 is involved in the biosynthesis of fatty acids and cholesterol and is mainly expressed in the liver and intestine [35]. In S1 mice, Acat2 carries one deleterious mutation that leads to a Valine/Methionine substitution at amino acid position 216 located in the conserved $\mathrm{N}$-terminal domain. This domain is important for the thiolase activity and a mutation in this region could have effects on the protein function. Thus, Acat2 is a good candidate for the observed hepatic fat storage in $\mathrm{S} 1$ mice.

Fmo5 and Notch2 were identified as the most likely candidate genes in Gatq1 affecting gonadal adipose tissue weight directly. S1 mice carry two SNPs in the promoter of Fmo5. According to the Ensembl database one SNP affects a transcription factor binding site for Elf5. The other SNP affects two transcription factor binding sites; one for Rxra and one for multiple transcription factors such as Nr2f6, Rara, Rarb, and Rarg. According to Bgee database, all identified transcription factors that potentially bind to the promoter region of Fmo5 are expressed in adipose tissue. The identified SNPs in the promoter of $\mathrm{Fmo5}$ could therefore be responsible for its lower expression in the gonadal adipose tissue and liver of S1 mice. Consistent with the QTL allele effect of S1 mice leading to lower adipose tissue weight and lower blood glucose concentration, Fmo5 knockout mice store less fat in gonadal adipose tissue and have lower blood glucose concentration at 20 weeks [36]. Notch2 is important for developmental processes by controlling cell fate decisions [37] and lipid storage [38]. Notch2 has been linked to type 2 diabetes in humans [39]. S1 mice carry a deleterious mutation leading to a Glycine/Serine substitution at amino acid position 136. This mutation resides in the EGF-like domain which is important for Notch2 activation [40] and could be causal for the low-fat deposition in gonadal adipose tissue found in S1 mice.

Based on the findings of this genetic study, additional research is necessary to further validate the suggested candidate genes. This could be done by knockout of certain genes, or through continuation of the AlL to reduce the physical length of QTL regions and thereby the number of candidate genes. It is important to note that, although we have prioritized candidate genes using all available information, we cannot completely rule out that one of the polymorphic genes or even an unannotated gene was wrongly discarded.

The human metabolic syndrome is a complex disease with many actors, many still unknown, contributing to its expression. The identification of new potential partners in the network by QTL analysis and subsequent data analysis could help to replenish the gaps.

\section{REFERENCES}

1. Aguilar-Salinas CA, Viveros-Ruiz T. Recent advances in managing/understanding the metabolic syndrome. F1000Research. 2019;8:370. Available from: https:// f1000research.com/articles/8-370/v1.

2. Goodarzi MO. Genetics of obesity: what genetic association studies have taught us about the biology of obesity and its complications. Lancet Diabetes Endocrinol. 2018;6:223-36. Available from: https://linkinghub.elsevier.com/retrieve/ pii/S2213858717302000.

3. Wagener A, Schmitt AO, Aksu S, Schlote W, Neuschl C, Brockmann GA. Genetic, sex, and diet effects on body weight and obesity in the Berlin Fat Mouse Inbred lines. Physiol Genom. 2006;27:264-70. Available from: http://www.ncbi.nlm.nih. gov/pubmed/16912068.

4. Neuschl C, Hantschel C, Wagener A, Schmitt AO, Illig T, Brockmann GA. A unique genetic defect on chromosome 3 is responsible for juvenile obesity in the Berlin Fat Mouse. Int J Obes. 2010;34:1706-14. Available from: http://www.ncbi.nlm.nih. gov/pubmed/20498659.

5. Arends D, Heise S, Kärst S, Trost J, Brockmann GA. Fine mapping a major obesity locus (jObes1) using a Berlin Fat Mouse $\times \mathrm{B} 6 \mathrm{~N}$ advanced intercross population. Int J Obes. 2016:40:1784-8. Available from: http://www.ncbi.nlm.nih.gov/pubmed/27538457.

6. Heise S, Trost J, Arends D, Wirth EK, Schäfer N, Köhrle J, et al. High variability of insulin sensitivity in closely related obese mouse inbred strains. Exp Clin Endocrinol Diabetes. 2016;124:519-28. Available from: http://www.ncbi.nlm.nih.gov/pubmed/ 27437914.

7. Schmitt AO, Bortfeldt R, Neuschl C, Brockmann GA. RandoMate: a program for the generation of random mating schemes for small laboratory animals. Mamm Genome. 2009;20:321-5. Available from: http://www.ncbi.nlm.nih.gov/pubmed/19444508.

8. Kluth $O$, Matzke $D$, Kamitz A, Jähnert $M$, Vogel $H$, Scherneck $S$, et al. Identification of four mouse diabetes candidate genes altering $\beta$-cell proliferation. Barsh GS, editor. PLOS Genet. 2015;11:e1005506. Available from: https://dx.plos.org/ 10.1371/journal.pgen.1005506.

9. Kluth O, Mirhashemi F, Scherneck S, Kaiser D, Kluge R, Neschen S, et al. Dissociation of lipotoxicity and glucotoxicity in a mouse model of obesity associated diabetes: role of forkhead box $\mathrm{O} 1$ (FOXO1) in glucose-induced beta cell failure. Diabetologia. 2011;54:605-16. Available from: http://www.ncbi.nlm.nih.gov/pubmed/21107520.

10. Hesse D, Trost J, Schäfer N, Schwerbel K, Hoeflich A, Schürmann A, et al. Effect of adipocyte-derived IGF-I on adipose tissue mass and glucose metabolism in the Berlin Fat Mouse. Growth Factors. 2018;36:78-88. Available from: https://www. tandfonline.com/doi/full/10.1080/08977194.2018.1497621.

11. Hesse $D$, Radloff $K$, Jaschke A, Lagerpusch M, Chung B, Tailleux A, et al. Hepatic trans-Golgi action coordinated by the GTPase ARFRP1 is crucial for lipoprotein lipidation and assembly. J Lipid Res. 2014;55:41-52. Available from: http://www. ncbi.nlm.nih.gov/pubmed/24186947.

12. Morgan AP, Fu C-P, Kao C-Y, Welsh CE, Didion JP, Yadgary $L$, et al. The mouse universal genotyping array: from substrains to subspecies. G3: Genes|Genomes| Genetics. 2016;6:263-79. Available from: https://academic.oup.com/g3journal/ article/6/2/263-279/6027250.

13. Kreuzer S, Reissmann M, Brockmann GA. Gene test to elucidate the ETEC F4ab/ F4ac receptor status in pigs. Vet Microbiol. 2013;162:293-5. Available from: https://linkinghub.elsevier.com/retrieve/pii/S037811351200452X.

14. Gao X. Multiple testing corrections for imputed SNPs. Genet Epidemiol. 2011;35:154-8. Available from: http://doi.wiley.com/10.1002/gepi.20563. 
15. Haynes W. Bonferroni correction. In: Encyclopedia of systems biology. New York, NY: Springer New York; 2013. p. 154-154. http://link.springer.com/10.1007/978-14419-9863-7_1213.

16. Dupuis J, Siegmund D. Statistical methods for mapping quantitative trait loci from a dense set of markers. Genetics. 1999;151:373-86. Available from: http:// www.ncbi.nlm.nih.gov/pubmed/9872974.

17. Li Y, Tesson BM, Churchill GA, Jansen RC. Critical reasoning on causal inference in genome-wide linkage and association studies. Trends Genet. 2010;26:493-8. Available from: https://linkinghub.elsevier.com/retrieve/pii/S0168952510001885.

18. Brockmann GA, Tsaih S-W, Neuschl C, Churchill GA, Li R. Genetic factors contributing to obesity and body weight can act through mechanisms affecting muscle weight, fat weight, or both. Physiol Genom. 2009;36:114-26. Available from: https://www.physiology.org/doi/10.1152/physiolgenomics.90277.2008.

19. Li H, Handsaker B, Wysoker A, Fennell T, Ruan J, Homer N, et al. The sequence alignment/map format and SAMtools. Bioinformatics. 2009;25:2078-9. Available from: https://academic.oup.com/bioinformatics/article-lookup/doi/10.1093/bioinformatics/ btp352.

20. McLaren W, Gil L, Hunt SE, Riat HS, Ritchie GRS, Thormann A, et al. The ensemble variant effect predictor. Genome Biol. 2016;17:122 Available from: http:// genomebiology.biomedcentral.com/articles/10.1186/s13059-016-0974-4.

21. Gotoh M, Maki T, Kiyoizumi T, Satomi S. Monaco Ap. An improved method for isolation of mouse pancreatic islets. Transplantation. 1985;40:437. Available from: http://journals.Iww.com/00007890-198510000-00018.

22. R Core Team (2018). R: a language and environment for statistical computing. $R$ Foundation for Statistical Computing, Vienna, Austria.

23. Livak KJ, Schmittgen TD. Analysis of relative gene expression data using real-time quantitative PCR and the $2-\Delta \Delta C T$ method. Methods. 2001;25:402-8. Available from: https://linkinghub.elsevier.com/retrieve/pii/S1046202301912629.

24. Durinck S, Spellman PT, Birney E, Huber W. Mapping identifiers for the integration of genomic datasets with the R/Bioconductor package biomaRt. Nat Protoc. 2009;4:1184-91. Available from: http://www.nature.com/articles/nprot.2009.97.

25. Kanehisa M. KEGG: Kyoto Encyclopedia of Genes and Genomes. Nucleic Acids Res. 2000;28:27-30. Available from: https://academic.oup.com/nar/article-lookup/ doi/10.1093/nar/28.1.27

26. Cava C, Bertoli G, Castiglioni I. Integrating genetics and epigenetics in breast cancer: biological insights, experimental, computational methods and therapeutic potential. BMC Syst Biol. 2015;9:62. Available from: https://bmcsystbiol. biomedcentral.com/articles/10.1186/s12918-015-0211-x.

27. Parker R. The role of adipose tissue in fatty liver diseases. Liver Res. 2018;2:35-42. Available from: https://linkinghub.elsevier.com/retrieve/pii/S2542568418000053.

28. Rosen ED, Spiegelman BM. Adipocytes as regulators of energy balance and glucose homeostasis. Nature. 2006;444:847-53. Available from: http://www. nature.com/articles/nature05483.

29. Ntountoumi C, Vlastaridis P, Mossialos D, Stathopoulos C, lliopoulos I, Promponas V, et al. Low complexity regions in the proteins of prokaryotes perform important functional roles and are highly conserved. Nucleic Acids Res. 2019;47:9998-10009. Available from: https://academic.oup.com/nar/article/47/19/9998/5559688.

30. Hoover-Plow J, Ellis J, Yuen L. In vivo plasminogen deficiency reduces fat accumulation. Thromb Haemost. 2002;87:1011-9. Available from: http://www.ncbi. nlm.nih.gov/pubmed/12083480.

31. Hoover-Plow J, Yuen L. Plasminogen binding is increased with adipocyte differentiation. Biochem Biophys Res Commun. 2001;284:389-94. Available from: https://linkinghub.elsevier.com/retrieve/pii/S0006291X01949848.

32. Ghosh AK, Vaughan DE. PAl-1 in tissue fibrosis. J Cell Physiol. 2012;227:493-507. Available from: http://doi.wiley.com/10.1002/jcp.22783.

33. Ajjan RA, Gamlen T, Standeven KF, Mughal S, Hess K, Smith KA, et al. Diabetes is associated with posttranslational modifications in plasminogen resulting in reduced plasmin generation and enzyme-specific activity. Blood. 2013;122:134-42. Available from: https://ashpublications.org/blood/article/122/1/134/31587/Diabetesis-associated-with-posttranslational.

34. Qi Q, Workalemahu T, Zhang C, Hu FB, Qi L. Genetic variants, plasma lipoprotein(a) levels, and risk of cardiovascular morbidity and mortality among two prospective cohorts of type 2 diabetes. Eur Heart J. 2012;33:325-34. Available from: https:// academic.oup.com/eurheartj/article-lookup/doi/10.1093/eurheartj/ehr350.

35. Fukao T, Song X-Q, Mitchell GA, Yamaguchi S, Sukegawa K, Or T, et al. Enzymes of ketone body utilization in human tissues: protein and messenger RNA levels of

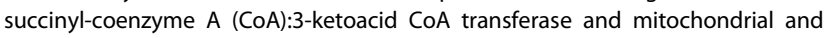
cytosolic acetoacetyl-CoA thiolases. Pediatr Res. 1997;42:498-502. Available from: http://www.nature.com/doifinder/10.1203/00006450-199710000-00013.

36. Gonzalez Malagon SG, Melidoni AN, Hernandez D, Omar BA, Houseman L, Veeravalli $S$, et al. The phenotype of a knockout mouse identifies flavin- containing monooxygenase 5 (FMO5) as a regulator of metabolic ageing. Biochem Pharmacol. 2015;96:267-77. Available from: https://linkinghub.elsevier. com/retrieve/pii/S0006295215002968.

37. Siebel C, Lendahl U. Notch signaling in development, tissue homeostasis, and disease. Physiol Rev. 2017;97:1235-94. Available from: https://www.physiology. org/doi/10.1152/physrev.00005.2017.

38. Bissonnette MLZ, Lane JC, Chang A. Extreme renal pathology in alagille syndrome. Kidney Int Rep. 2017;2:493-7. Available from: https://linkinghub.elsevier. com/retrieve/pii/S246802491630167X.

39. Zeggini E, Scott LJ, Saxena R, Voight BF, Marchini JL, Hu T, et al. Meta-analysis of genome-wide association data and large-scale replication identifies additional susceptibility loci for type 2 diabetes. Nat Genet. 2008;40:638-45. Available from: http://www.nature.com/articles/ng.120.

40. Lai EC. Notch signaling: control of cell communication and cell fate. Development. 2004;131:965-73. Available from: http://dev.biologists.org/cgi/doi/10.1242/dev.01074.

\section{ACKNOWLEDGEMENTS}

The project was funded by the Deutsche Forschungsgemeinschaft (DFG, HE8165/1-1). We thank Marion Bütow, Ulf Kiessling, and Ines Walter for assistance in animal care. We also thank Anett Helms and Franziska Gabler for pancreatic islet isolation and RNA extraction.

\section{AUTHOR CONTRIBUTIONS}

MD: Data collection, data analysis, and manuscript draft. DA: Method discussion, data analysis, and manuscript revision. MS: Data collection. FK: Data collection. OK: Islet isolation, and data collection. AS: Method discussion and manuscript feedback. GAB: Data discussion, supervision, and manuscript feedback. DH: Experimental design, supervision, method discussion, and manuscript draft, feedback, and revision.

\section{FUNDING}

Open Access funding enabled and organized by Projekt DEAL.

\section{COMPETING INTERESTS}

The authors declare no competing financial interests in relation to the work described.

\section{ADDITIONAL INFORMATION}

Supplementary information The online version contains supplementary material available at https://doi.org/10.1038/s41366-021-00991-3.

Correspondence and requests for materials should be addressed to Deike Hesse.

Reprints and permission information is available at http://www.nature.com/ reprints

Publisher's note Springer Nature remains neutral with regard to jurisdictional claims in published maps and institutional affiliations.

\footnotetext{
Open Access This article is licensed under a Creative Commons Attribution 4.0 International License, which permits use, sharing, adaptation, distribution and reproduction in any medium or format, as long as you give appropriate credit to the original author(s) and the source, provide a link to the Creative Commons license, and indicate if changes were made. The images or other third party material in this article are included in the article's Creative Commons license, unless indicated otherwise in a credit line to the material. If material is not included in the article's Creative Commons license and your intended use is not permitted by statutory regulation or exceeds the permitted use, you will need to obtain permission directly from the copyright holder. To view a copy of this license, visit http://creativecommons. org/licenses/by/4.0/.
}

(c) The Author(s) 2021 